\title{
Double immunolabeling of central nervous system atypical teratoid/rhabdoid tumors
}

\author{
John-Paul Bouffard ${ }^{1}$, Glenn D Sandberg ${ }^{1}$, Jeffrey A Golden² and Lucy B Rorke ${ }^{2}$ \\ ${ }^{1}$ Department of Neuropathology \& Ophthalmic Pathology, Armed Forces Institute of Pathology, Washington, \\ DC, USA and ${ }^{2}$ Department of Pathology, Children's Hospital of Philadelphia, Philadelphia, PA, USA
}

\begin{abstract}
The central nervous system atypical teratoid/rhabdoid tumor (ATRT) is a highly malignant tumor with a heterogeneous immunohistochemical profile and with some morphologic similarity to central nervous system primitive neuroectodermal tumors (PNET). Although several studies have investigated double immunolabeling in PNET, we are aware of no studies of double labeling of ATRT. A total of 10 ATRT from surgical and consultation materials at the Children's Hospital of Philadelphia were selected and stained for a variety of antigens using indirect immunofluorescence to detect single and double labeling. Most tumor cells showed only single labeling; rare cells showed double labeling as follows: $\mathbf{7 0} \%$ of tumors coexpressed (VIM) and glial fibrillary acidic protein (GFAP), 30\% smooth muscle actin and GFAP, $20 \%$ epithelial membrane antigen (EMA) and VIM, 20\% EMA/GFAP, and 20\% EMA/SMA. These results are discussed in view of current debates over the histogenesis of CNS PNET and ATRT, and in reference to the classification of rhabdoid tumors as an entity or phenotype.

Modern Pathology (2004) 17, 679-683, advance online publication, 23 April 2004; doi:10.1038/modpathol.3800099
\end{abstract}

Keywords: rhabdoid; central nervous system; primitive neuroectodermal tumor

The central nervous system atypical teratoid/rhabdoid tumor (ATRT), first described by Rorke et al in $1987^{1}$ is a highly malignant tumor occurring primarily in infants and children. The histologic and immunohistochemical features of this neoplasm have been extensively described. ${ }^{2-5}$ It is composed partially or entirely of large, highly pleomorphic "rhabdoid" cells, often with filamentous cytoplasmic inclusions, which express one or more of a heterogeneous variety of antigens of neural (neurofilament protein (NFP), glial fibrillary acidic protein (GFAP), synaptophysin), mesenchymal (smooth muscle actin (SMA), vimentin (VIM), and epithelial (epithelial membrane antigen (EMA), cytokeratin) derivation. The presence of areas histologically similar to primitive neuroectodermal tumor (PNET) in most ATRTs has raised questions about the relationship between these two neoplasms. PNETs are known to express NFP, synaptophysin, VIM, desmin, and, in some cases, cytokeratin, and GFAP, and in double and triple labeling studies have been shown to coexpress some of these antigens, ${ }^{6-8}$ but we know of no studies investigating double

Correspondence: John-Paul Bouffard, MD, LtCol, USAF MC, Armed Forces Institute of Pathology, 6825 16th Street NW, Building 54, Room G051, Washington, DC 20206-6000, USA.

E-mail: bouffardj@afip.osd.mil

Received 2 May 2003; revised 31 October 2003; accepted 5 January 2004; published online 23 April 2004 immunolabeling of rhabdoid tumors. The purpose of this study was to evaluate a group of ATRT for coexpression of a variety of these antigens.

\section{Materials and methods}

Ten cases of ATRT were chosen from surgical and consultation material at the Children's Hospital of Philadelphia between 1987 and 1998. Diagnoses were made from hemotoxylin and eosin (H\&E) sections prepared from standard formalin-fixed, paraffin-embedded tissues, and with a variety of immunohistochemical stains (standard avidin-biotin immunoperoxidase (IP) method). All cases were studied for expression of NFP, GFAP, VIM, EMA and SMA. Additional sections prepared from the paraffin blocks were also studied by immunofluoresence (IF) microscopy for all of these antigens and for dual expression of NFP/GFAP, VIM/GFAP, SMA/GFAP, EMA/GFAP, EMA/NFP, EMA/SMA and EMA/VIM. Although EMA is known to be a marker for both epithelial and nonepithelial tissues (perineurium, mesothelium, plasma cells, etc.), it was chosen as the epithelial marker in this case to avoid issues of cross reactivity associated with pancytokeratin (ie, with GFAP). Fluorochromes used were fluorescein isothiocyanate (FITC) and tetramethyl rhodamine isothiocyanate (TRITC), and 4',6-diamidino-2-phenylindole (DAPI), all commercially obtained (see the 
appendix). Appropriate dilution studies were conducted to obtain the best possible fluorescent staining with the least amount of background.

Expression of antigens by IP was determined by observing the standard brown, horseradish peroxidase reaction product against the blue counterstain. For IF studies, expression of a single antigen was determined by observing either a green (FITC), or red (TRITC), reaction product in the tumor cells. DAPI (blue color) was used as a nuclear counterstain in some cases. Dual antigen expression in single cells (double labeling) was determined by observing a yellow or light orange (FITC + TRITC) reaction product in the tumor cells.

\section{Results}

Expression of the various antigens was scored using a semiquantitative scale (1-least through 4-greatest) based on the intensity of the reaction and the proportion of cells stained in the sections examined. Data collection was focused on ascertaining antigen expression in rhabdoid cells, using correlative H\&E-stained sections to select those fields for fluorescent microscopy in which rhabdoid cells formed the predominant pattern. Results are summarized in Tables 2 and 3 . The vast majority of cells in all tumors showed only single immunolabeling; double labeling was the exception. By standard immunoperoxidase, six of 10 tumors expressed NFP, seven of 10 expressed GFAP, 10 of 10 expressed vimentin, eight of 10 expressed EMA, and eight of 10 expressed SMA. By immunofluorescence, four of 10 cases expressed NFP, nine of 10 expressed GFAP, 10 of 10 expressed VIM, seven of 10 expressed SMA, and five of 10 expressed EMA. Eight of 10 cases showed coexpression of at least one antigen pair; one case showed at least focal coexpression of five of the six antigen pairs tested. Overall, the most common coexpressed antigens were VIM/GFAP (seven of 10 cases). There was coexpression of SMA/GFAP in three of 10 cases, and EMA/VIM in two of 10 cases. Three tumors showed focal coexpression of GFAP/ EMA, and two cases showed focal coexpression of EMA/SMA. All coexpression involving EMA and

Table 1 Patient data

\begin{tabular}{clll}
\hline Case & Age & Gender & Location \\
\hline 1 & 11 months & Male & Posterior fossa \\
2 & 16 months & Female & Posterior fossa \\
3 & 3 years & Male & Frontal lobe \\
4 & 9 months & Male & Pineal region \\
5 & 10 months & Female & Pons \\
6 & 1 year & Male & Posterior fossa \\
7 & 10 years & Female & Posterior fossa \\
8 & 6 years & Female & Left frontal lobe \\
9 & 5 years & Male & Left frontal lobe \\
10 & 10 years & Male & Cerebellum \\
\hline
\end{tabular}

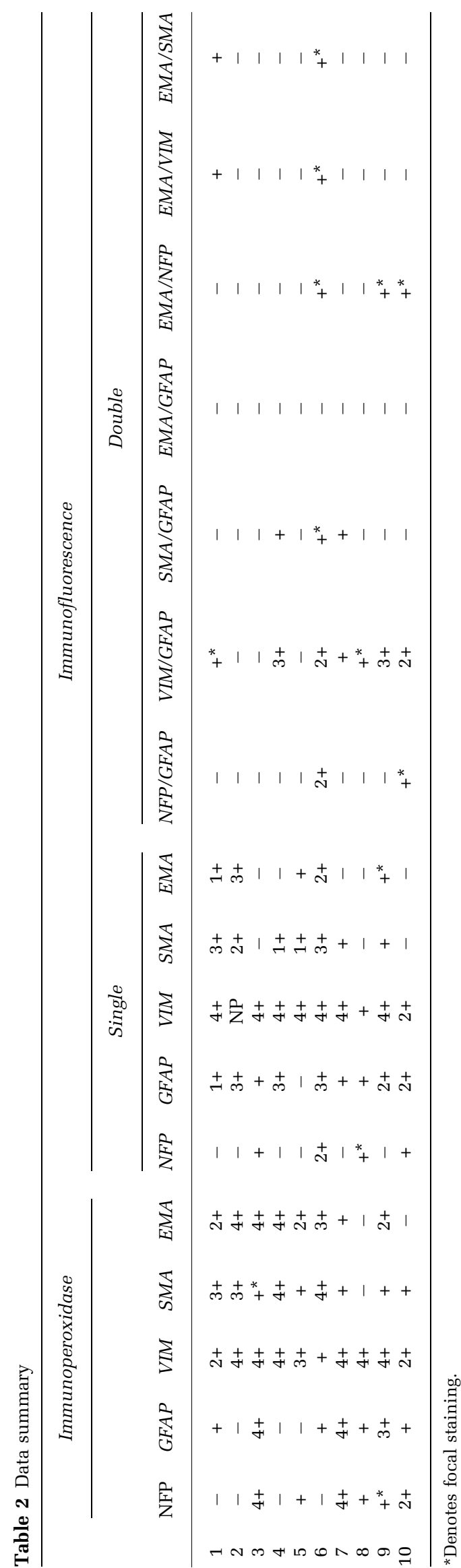


Table 3 Proportion of cases with single or dual expression by immunofluorescence

\begin{tabular}{llllll}
\hline & \multirow{2}{*}{ NFP } & \multirow{2}{*}{ GFAP } & VIM & SMA & EMA \\
\hline NFP & \multirow{2}{*}{$4 / 10$} & $2 / 10$ & NP & NP & $0 / 10$ \\
GFAP & & $9 / 10$ & $7 / 10$ & $3 / 10$ & $3 / 10$ \\
VIM & & & $9 / 9$ & NP & $2 / 10$ \\
SMA & & & & $7 / 10$ & $2 / 10$ \\
EMA & & & & & $5 / 10$
\end{tabular}

$\mathrm{NP}=$ not performed

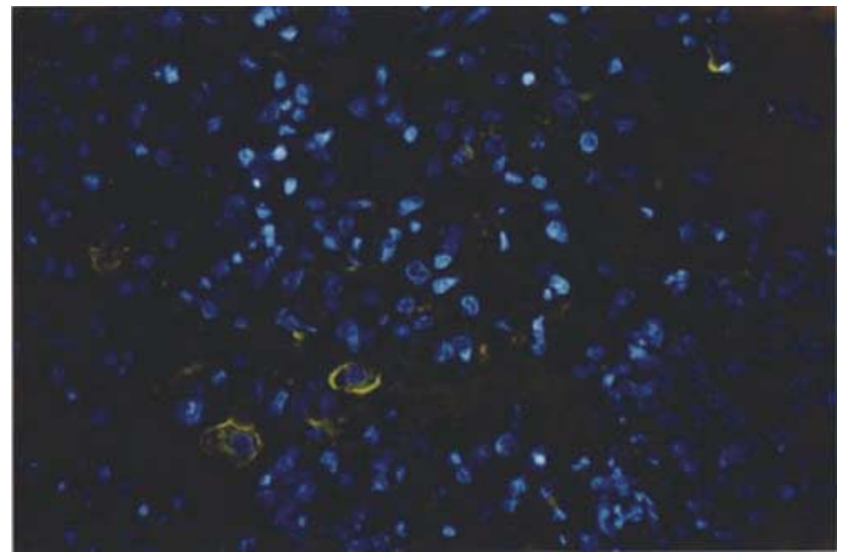

Figure 1 Double immunolabeling (yellow/light orange fluorescence) for EMA and GFAP. Note rim pattern of staining. Blue (DAPI) staining identifies cell nuclei $\times 40$.

other antigens occurred in rhabdoid cells, and often showed a cytoplasmic rim pattern of reactivity (Figure 1). Coexpression of VIM/GFAP was observed in both rhabdoid and nonrhabdoid cells.

Differences in immunophenotype as determined by IP vs IF methods (Table 2) are largely attributable to sampling differences between the two methods. IF determinations were restricted to fields of rhabdoid cells, while IP determinations were not so restricted.

Photomicrographs of representative immunofluorescence stains are provided in Figures 1 and 2. A brief summary of patient data is included in Table 1. Follow-up data were not obtained.

\section{Discussion}

The difficulty of classifying and understanding a tumor composed of subpopulations of cells with widely divergent morphology and antigen expression is now compounded by finding such divergence within individual cells. Particularly noteworthy in this regard is the finding of cells (although only in rare cells in two cases) with coexpression of mature epithelial and glial antigens (EMA/GFAP). Generally speaking, these data confirm what has already been suggested about the ATRT: rhabdoid cells do strange, counterintuitive

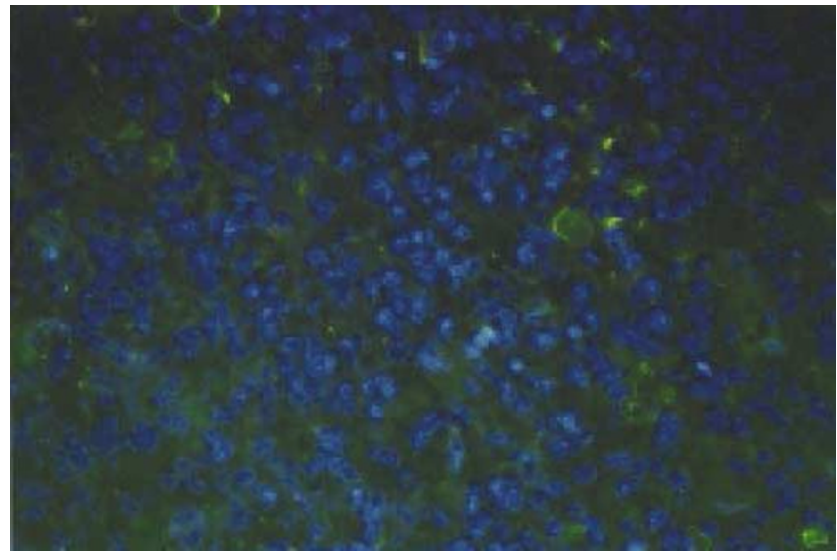

Figure 2 Double immunolabeling (yellow) for EMA and SMA. Blue (DAPI) staining identifies cell nuclei $\times 40$.

things, and do not fit neatly into any classification system based on tumor cell lineage. Although the most promising path toward ascertaining the true nature of the ATRT is probably paved by research in molecular genetics, these immunohistochemical data do invite fruitful discussion relevant to some facets of the problem.

The first question that must be addressed in interpreting these results is whether the immunoreactivity observed could be in any way spurious. It has been suggested that in the case of rhabdoid cells, the cytoplasmic filamentous inclusions may be capable of 'trapping' antigens in a nonspecific manner, a hypothesis that offers a simple explanation of anomalous antigen expression. ${ }^{9}$ In our study, however, double labeling of multiple pairs of antigens, including EMA/GFAP, was observed either diffusely in the cytoplasm or in a subplasmalemmal rim pattern, and not in typical cytoplasmic inclusions. Appropriate positive and negative controls permit exclusion of other types of nonspecific binding.

The pattern of multiple immunolabeling observed in these tumors suggests that most individual tumor cells remain 'faithful' to a single antigenic class, with double expression being the exception rather than the rule. Double-labeled cells were often single cells, set apart from 'clonal' groups of cells expressing one of the various other antigens tested. Compared to double-labeling studies of PNET, ${ }^{6-8}$ the ATRT in this study show less double labeling. We find this difference interesting, given the fact that most ATRT contain areas of classical PNET. One hypothesis for the histogenesis of ATRT is malignant transformation of a putative pluripotent stem cell-a more primitive precursor than that of PNET. One might therefore expect a neoplasm with such a pluripotent origin to show more antigenic heterogeneity than PNET, but the opposite appears true.

Since the first descriptions of rhabdoid tumor of the kidney (RTK), ${ }^{10,11}$ CNS ATRT, ${ }^{1-3}$ and extra-renal 
rhabdoid tumors (ERRT), ${ }^{4,12-14}$ there has been considerable debate over whether rhabdoid tumors constitute a unique entity or are merely a variant/ phenotype of many different tumors. The concept of the rhabdoid tumor as an entity, as it has been couched in this debate, requires a putative, unique progenitor cell common to all rhabdoid tumors, irrespective of their location in the body, while the concept of a rhabdoid phenotype suggests, as eloquently stated by Wick et $a l^{4}$ 'a common end point of clonal evolution in tumors of clearly different origin' (italics added, 3). While Rorke, in discussing PNET, ${ }^{15-17}$ has questioned both the practical utility and the logical premises underlying debates on the concept of 'cell of origin,' the data in this study are relevant in this regard and merit discussion.

Given the well-established heterogeneity of antigen expression in ATRT, and accepting the hypothesis that malignant tumors represent transformation of a putative precursor cell by one or more genetic mutations which both immortalize and confer invasive capability, the concept of rhabdoid tumor as an entity suggests (but does not necessarily require) the existence of a precursor cell capable of differentiation along multiple, dissimilar lineages. A neoplasm evolving from such a pluripotent precursor would be expected to contain at least some cells which coexpress seemingly illogical combinations of antigens, as observed in this study. An analogous situation has been observed in CNS PNET. There is credible evidence that CNS PNET arise from precursor cells capable of differentiation along both glial and neuronal lines, ${ }^{18-21}$ and double-labeling studies have demonstrated coexpression of not only glial and neuronal antigens in these tumors, ${ }^{6,7}$ but also of other types of intermediate filament antigens such as VIM, desmin, and keratin. Inferences made from the age distribution of CNS ATRT also support the concept of a pluripotent precursor cell. The early, often neonatal presentation of this tumor indicates that it often forms in utero, when there are almost certainly many more undifferentiated, pluripotent cells present than later in life. Furthermore, the microenvironment of a developing fetus must harbor active signaling mechanisms required for lineage-specific cell differentiation. Thus, it is logical to assume that a neoplasm evolving from pluripotent cells might respond to such an environment with peculiar patterns of differentiation.

Yet there is also evidence supporting the phenotype concept. One such observation is the so-called composite extrarenal rhabdoid tumor (CERT; 11). These are tumors composed of a 'collision' of a welldefined neoplasm such as a carcinoma, sarcoma, or melanoma, with a proliferation of malignant rhabdoid cells. Numerous examples have been reported, involving such neoplasms as endometrial carcinoma, melanoma, and meningioma. ${ }^{19,22}$ In the series reported by Fuller et $a l,{ }^{14}$ this subset of rhabdoid tumors is distinguished from CNS ATRT and RTK by a significantly lower incidence of abnormalities of chromosome 22q, and they propose that CERTs originate as common tumors and 'evolve' a rhabdoid clonal population. Following this line of reasoning, one could interpret CNS ATRT (particularly those without chromosome 22q abnormalities) as CERTs, with PNET playing the role of 'parent' neoplasm. Although the widely divergent molecular genetic profiles of PNET and ATRT (abnormality of chromosome 22 in ATRT $^{2}$ vs isochromosome 17q in PNET $^{16}$ ) are compelling evidence that these tumors are separate, distinct entities, it should be considered that at least some CNS ATRT might represent variations of, or have evolved from, an otherwise typical PNET. The pattern of double labeling observed in this study overlaps with that reported by Gould et $a l^{7}$ for PNET, ${ }^{6}$ suggesting just such a possibility.

For practical purposes, the debate over the nature of rhabdoid cells may be nothing more than a semantic exercise, since the most homogeneous characteristic of this otherwise heterogeneous group of tumors is the almost universally grim prognosis, wherever and whenever they occur. If tumors in different locations display similar biologic behavior, does it matter whether the similarity is a manifestation of a common progenitor cell or a common molecular genetic event? At the moment, the answer seems to be no. But if there is any hope of developing effective treatments for these tumors, these intellectual exercises, and the research they inspire, must continue.

\section{References}

1 Lefkowitz IB, Rorke LB, Packer RJ. Atypical teratoid tumor of infancy: definition of an entity. Ann Neurol 1987;22:448-449 (abstract).

2 Rorke LB, Packer RJ, Biegel JA. Central nervous system atypical teratoid/rhabdoid tumors of infancy and childhood: definition of an entity. J Neurosurg 1996;85:56-65.

3 Rorke LB, Packer RJ, Biegel JA. Central nervous system atypical teratoid/rhabdoid tumors of infancy and childhood. J Neuro-oncol 1995;24:21-28.

4 Wick MR, Ritter JH, Dehner LP. Malignant rhabdoid tumors: a clinicopathologic review and conceptual discussion. Semin Diagn Pathol 1995;12(3):233-248.

5 Weeks DA, Beckwith JB, Mierau GW. Rhabdoid tumor: an entity or a phenotype? Arch Pathol Lab Med 1989;113:113-114.

6 Molenaar WM, Jansson DS, Gould VE, et al. Molecular markers of primitive neuroectodermal tumors and other pediatric central nervous system tumors. Monoclonal antibodies to neuronal and glial antigens distinguish subsets of primitive neuroectodermal tumors. Lab Invest 1989;61(6):635-643.

7 Gould VE, Jansson DS, Molenaar WM, et al. Primitive neuroectodermal tumors of the central nervous system. Patterns of expression of neuroendocrine markers, and all classes of intermediate filament proteins. Lab Invest 1990;62(4):498-509. 
8 Gould VE, Rorke LB, Jansson DS, et al. Primitive neuroectodermal tumors of the central nervous system express neuroendocrine markers and may express all classes of intermediate filaments. Hum Pathol 1990;24(3):245-252.

9 Weeks DA, Beckwith JB, Mierau GW, et al. Rhabdoid tumor of the kidney. A report of 111 cases from the national Wilms' tumor study pathology center. Am J Surg Pathol 1989;13(6):439-458.

10 Beckwith JB, Palmer NF. Histopathology and prognosis of Wilms' tumor. Results from the first National Wilms' Tumor study. Cancer 1978;41:1937-1948.

11 Beckwith JB. Wilms' tumor and other renal tumors of childhood: a selective review from the National Wilms' Tumor Study Pathology Center. Hum Pathol 1983;14(6):481-492.

12 Tsokos M, et al. Malignant rhabdoid tumor of the kidney and soft tissues. Arch Pathol Lab Med 1989;113:115-120.

13 Knapik J, Yachnis AT, Ripley D, et al. Aggressive uterine sarcoma with rhabdoid features: diagnosis by peritoneal fluid cytology and absence of INI-1 gene mutation. Hum Pathol 2001;32(8):884-886.

14 Fuller CE, Pfeifer J, Humphrey P, et al. Chromosome $22 q$ dosage in composite extrarenal rhabdoid tumors: clonal evolution or a phenotypic mimic? Hum Pathol 2001;32(10):1102-1108.

15 Rorke LB. The cerebellar medulloblastoma and its relationship to primitive neuroectodermal tumors. J Neuropathol Exp Neurol 1983;42(1):1-15.

16 Rorke LB, Trojanowski JQ, Lee VM, et al. Primitive neuroectodermal tumors of the central nervous system. Brain Pathol 1997;7:765-784.

17 Rorke LB. Experimental production of primitive neuroectodermal tumors and its relevance to human neuro-oncology. Am J Pathol 1994;44(3):444-448.

18 Friedman HS, Burger PC, Bigner SH, et al. Phenotypic and genotypic analysis of human medulloblastoma cell line and transplantable xenograft (D341 Med) demonstrating amplifications of c-myc. Am J Pathol 1988;130:472-484.

19 Friedman HS, Burger PC, Bigner SH, et al. Establishment and characterization of the human medulloblastoma cell line and transplantable xeno- graft D283 MED. J Neuropathol Exp Neurol 1985;44:592-605.

20 Trojanowski JQ, Friedman HS, Burger PC, et al. Rapidly dividing human medulloblastoma cell line (D283 MED) expresses all three neurofilament subunits. Am J Pathol 1987;126:358-363.

21 He XM, Skapek SX, Wikstrand CJ, et al. Phenotypic analysis of four human medulloblastoma cell lines and transplantable xenografts. J Neuropathol Exp Neurol 1989;48:48-68.

22 Gaertner EM, Farley JH, Taylor RR, et al. Collision of uterine rhabdoid tumor and endometrioid adenocarcinoma: a case report and review of the literature. Int J Gynecol Pathol 1999;18(4):396-401.

\section{Appendix: Fluorescent antibodies}

(1) Rhodamine (TRITC)-conjugated AffiniPure Donkey Anti-Sheep IgG, Code number 713025-147. Jackson Immuno Research Laboratories, Inc.

(2) Fluorescein (FITC) also obtained from Jackson ImmunoResearch Laboratories, Inc.

(3) Monoclonal anti-glial fibrillary acidic protein (GFAP) Cy3 conjugate, product no. C-9205, Sigma Chemical Company.

(4) Sheep anti-EMA, Cat. No. 64-736, ICN Biomedicals.

(5) Monoclonal mouse anti-human alpha-SMA, Clone 1A4, Code no. M 0851, DAKO.

(6) Monoclonal mouse anti-VIM, Clone V9, code no. M 0725, DAKO.

(7) Monoclonal mouse anti-neurofilament proteins, middle and high molecular weight, clone RmdO-20, Zymed Laboratories, Inc.

(8) Normal donkey serum, code number 017-000121, used for blocker, Jackson ImmunoResearch Laboratories, Inc. 